PROCEEDINGS OF THE

AMERICAN MATHEMATICAL SOCIETY

Volume 131, Number 2, Pages 379-387

S 0002-9939(02)06326-8

Article electronically published on September 17, 2002

\title{
SOME RESULTS ON EXTREMAL VECTORS AND INVARIANT SUBSPACES
}

\author{
PER ENFLO AND TERJE HÕIM
}

(Communicated by N. Tomczak-Jaegermann)

\begin{abstract}
In 1996 P. Enflo introduced the concept of extremal vectors and their connection to the Invariant Subspace Problem. The study of backward minimal vectors gives a new method of finding invariant subspaces which is more constructive than the previously known methods. In this article we study the properties and behaviour of extremal vectors, give some new formulas related to backward minimal vectors and improve results from papers by Ansari and Enflo (1998) and Enflo (1998).
\end{abstract}

In 1996 P.Enflo introduced a new method to find invariant subspaces using backward minimal vectors. This method gives hyperinvariant subspaces for all compact and all normal operators in a unified way. Studying a general bounded linear operator $T$ on Hilbert space and extremal vectors associated with the operator $T$, several parameters are coming up naturally. In order to further use extremal vectors to find invariant subspaces it seems important to know more about the connections between these parameters. In this article we study the properties and behaviour of extremal vectors, investigate the connections between many of the parameters that come up in this study, give some new formulas related to backward minimal vectors and improve results from [1] and [2].

We start by recalling the definition of backward minimal vectors from AnsariEnflo [1]. $H$ will denote a separable Hilbert space over the real or complex numbers. $R(T)$ will denote the range of $T$.

Definition 1. Let $T: H \rightarrow H$ be a bounded operator with dense range. Let $x_{0} \in H, x_{0} \notin R(T)$, and $\varepsilon>0$ with $\varepsilon<\left\|x_{0}\right\|$. There is a unique vector $y_{n, x_{0}}^{\varepsilon}$ such that $\left\|T^{n} y_{n, x_{0}}^{\varepsilon}-x_{0}\right\| \leq \varepsilon$ and

$$
\left\|y_{n, x_{0}}^{\varepsilon}\right\|=\inf \left\{\|y\|:\left\|T^{n} y-x_{0}\right\| \leq \varepsilon\right\} .
$$

The points $y_{n, x_{0}}^{\varepsilon}$ are called backward minimal points.

When there is no ambiguity, we will drop $x_{0}$ and $\varepsilon$ in $y_{n, x_{0}}^{\varepsilon}$. It is clear that $\left\|T^{n} y_{n, x_{0}}^{\varepsilon}-x_{0}\right\|=\varepsilon$.

Received by the editors August 15, 2000 and, in revised form, February 7, 2001.

2000 Mathematics Subject Classification. Primary 47A15.

Key words and phrases. Invariant subspaces, extremal vectors.

The first author was supported by an NSF grant. 
In [1] the following orthogonality equation is given:

Theorem 2 (S.Ansari, P.Enflo, [1]). There exists a constant $\delta_{\varepsilon}<0$ such that

$$
y_{\varepsilon}=\delta_{\varepsilon} T^{*}\left(T y_{\varepsilon}-x_{0}\right) \text {. }
$$

From this it follows that $T^{n} y_{n}=-\delta_{n}\left(I-\delta_{n} T^{n} T^{* n}\right)^{-1} T^{n} T^{* n} x_{0}$ for every $n$. The minimality of $y_{n, x_{0}}^{\varepsilon}=y_{n}$ and the previous theorem also give the following:

\section{Orthogonality Relation.}

$$
\text { If } r_{n} \perp y_{n} \text {, then } T^{n} r_{n} \perp T^{n} y_{n}-x_{0} .
$$

We will consider the function $\varepsilon \longmapsto\left\|y_{\varepsilon}\right\|$ and prove the following theorem.

Theorem 3. The function $\varepsilon \longmapsto\left\|y_{\varepsilon}\right\|$ is convex on $\left(0,\left\|x_{0}\right\|\right)$.

Proof. For any $\lambda \in[0,1]$ and for any $\varepsilon_{1}>0$ and $\varepsilon_{2}>0$ with $\varepsilon_{2}<\varepsilon_{1}$, let $\varepsilon_{\lambda}=\lambda \varepsilon_{1}+(1-\lambda) \varepsilon_{2}$. Since the element $\lambda T y_{\varepsilon_{1}}+(1-\lambda) T y_{\varepsilon_{2}}=T\left(\lambda y_{\varepsilon_{1}}+(1-\lambda) y_{\varepsilon_{2}}\right)$ is inside the ball around $x_{0}$ with radius $\varepsilon_{\lambda}$, by the minimality of $\left\|y_{\varepsilon_{\lambda}}\right\|$ we have $\left\|y_{\varepsilon_{\lambda}}\right\| \leq\left\|\lambda y_{\varepsilon_{1}}+(1-\lambda) y_{\varepsilon_{2}}\right\| \leq \lambda\left\|y_{\varepsilon_{1}}\right\|+(1-\lambda)\left\|y_{\varepsilon_{2}}\right\|$. Hence, $\varepsilon \longmapsto\left\|y_{\varepsilon}\right\|$ is convex.

Next we present the following new formula for the derivative of the function $\varepsilon \longmapsto\left\|y_{\varepsilon}\right\|$.

Theorem 4. For the function $f(\varepsilon)=\left\|y_{\varepsilon}\right\|$ on $\left(0,\left\|x_{0}\right\|\right)$ we have

$$
\frac{d}{d \varepsilon} f(\varepsilon)=\frac{\left\|y_{\varepsilon}\right\|}{\cos \theta_{\varepsilon}\left\|T y_{\varepsilon}\right\|},
$$

where $\theta_{\varepsilon}$ is the angle between the vectors $T y_{\varepsilon}-x_{0}$ and $T y_{\varepsilon}$.

Proof. Let $\varepsilon>0$ and let $h>0$. Consider the balls around $x_{0}$ with radii $\varepsilon$ and $\varepsilon-h$. Let $y_{\varepsilon}$ and $y_{\varepsilon-h}$ be the backward minimal points for these balls, respectively. Consider the plane spanned by $x_{0}$ and $T y_{\varepsilon}$. Since $\theta_{\varepsilon}>\frac{\pi}{2}$, there exist $t>0$ and $s>0$ such that $\left\|x_{0}-(1+t) T y_{\varepsilon}\right\|=\varepsilon \sin \theta_{\varepsilon}$ and $\left\|x_{0}-(1+s) T y_{\varepsilon}\right\|=\varepsilon-h$. From the Pythagorean Theorem,

$$
\left\|(1+t) T y_{\varepsilon}-(1+s) T y_{\varepsilon}\right\|=\sqrt{(\varepsilon-h)^{2}-\varepsilon^{2} \sin ^{2} \theta_{\varepsilon}},
$$

and thus

$$
\left\|(1+s) T y_{\varepsilon}-T y_{\varepsilon}\right\|=\varepsilon \cos \left(\pi-\theta_{\varepsilon}\right)-\sqrt{(\varepsilon-h)^{2}-\varepsilon^{2} \sin ^{2} \theta_{\varepsilon}} .
$$

Since $\left\|T y_{\varepsilon}\right\|=\sqrt{1-\varepsilon^{2} \sin ^{2} \theta_{\varepsilon}}-\varepsilon \cos \left(\pi-\theta_{\varepsilon}\right)$, we have by the minimality of $\left\|y_{\varepsilon-h}\right\|$ that

$$
\left\|y_{\varepsilon-h}\right\| \leq\left(1+\frac{\varepsilon \cos \left(\pi-\theta_{\varepsilon}\right)-\sqrt{(\varepsilon-h)^{2}-\varepsilon^{2} \sin ^{2} \theta_{\varepsilon}}}{\sqrt{1-\varepsilon^{2} \sin ^{2} \theta_{\varepsilon}}-\varepsilon \cos \left(\pi-\theta_{\varepsilon}\right)}\right)\left\|y_{\varepsilon}\right\| .
$$

On the other hand, if $T y_{\varepsilon-h}=a T y_{\varepsilon}+T r$, where $T r \perp x_{0}-T y_{\varepsilon}$ and $a>1$, then

$$
\left\|a T y_{\varepsilon}-T y_{\varepsilon}\right\|=\frac{h}{\cos \left(\pi-\theta_{\varepsilon}\right)},
$$

and thus

$$
\left(1+\frac{h}{\cos \left(\pi-\theta_{\varepsilon}\right)\left(\sqrt{1-\varepsilon^{2} \sin ^{2} \theta_{\varepsilon}}-\varepsilon \cos \left(\pi-\theta_{\varepsilon}\right)\right)}\right)\left\|y_{\varepsilon}\right\| \leq\left\|y_{\varepsilon-h}\right\| .
$$


So, we get that

$$
\begin{gathered}
\frac{\left\|y_{\varepsilon}\right\|}{\cos \left(\pi-\theta_{\varepsilon}\right)\left(\sqrt{1-\varepsilon^{2} \sin ^{2} \theta_{\varepsilon}}-\varepsilon \cos \left(\pi-\theta_{\varepsilon}\right)\right)} \leq \frac{\left\|y_{\varepsilon-h}\right\|-\left\|y_{\varepsilon}\right\|}{h} \\
\leq \frac{\varepsilon \cos \left(\pi-\theta_{\varepsilon}\right)-\sqrt{\varepsilon^{2}-2 \varepsilon h+h^{2}-\varepsilon^{2} \sin ^{2} \theta}}{h\left(\sqrt{1-\varepsilon^{2} \sin ^{2} \theta_{\varepsilon}}-\varepsilon \cos \left(\pi-\theta_{\varepsilon}\right)\right)}\left\|y_{\varepsilon}\right\| .
\end{gathered}
$$

Letting $h \rightarrow 0$ in the right-hand side of the inequality, we get

and thus

$$
\begin{aligned}
& \lim _{h \rightarrow 0} \frac{\varepsilon \cos \left(\pi-\theta_{\varepsilon}\right)-\sqrt{\varepsilon^{2}\left(1-\sin ^{2} \theta_{\varepsilon}\right)-2 \varepsilon h+h^{2}}}{h\left(\sqrt{1-\varepsilon^{2} \sin ^{2} \theta_{\varepsilon}}-\varepsilon \cos \left(\pi-\theta_{\varepsilon}\right)\right)} \\
& =\frac{1}{\cos \left(\pi-\theta_{\varepsilon}\right)\left(\sqrt{1-\varepsilon^{2} \sin ^{2} \theta_{\varepsilon}}-\varepsilon \cos \left(\pi-\theta_{\varepsilon}\right)\right)},
\end{aligned}
$$

$$
\begin{gathered}
-f^{\prime}(\varepsilon)=\lim _{h \rightarrow 0} \frac{\left\|y_{\varepsilon-h}\right\|-\left\|y_{\varepsilon}\right\|}{h} \\
=\frac{\left\|y_{\varepsilon}\right\|}{\cos \left(\pi-\theta_{\varepsilon}\right)\left(\sqrt{1-\varepsilon^{2} \sin ^{2} \theta_{\varepsilon}}-\varepsilon \cos \left(\pi-\theta_{\varepsilon}\right)\right)}=\frac{\left\|y_{\varepsilon}\right\|}{\cos \left(\pi-\theta_{\varepsilon}\right)\left\|T y_{\varepsilon}\right\|} .
\end{gathered}
$$

If $\theta_{\varepsilon}$ is close to $\frac{\pi}{2}$ and $\varepsilon>0$ is small, then $\left\|T y_{\varepsilon}\right\|=\sqrt{1-\varepsilon^{2} \sin ^{2} \theta_{\varepsilon}}-\varepsilon \cos \left(\pi-\theta_{\varepsilon}\right)$ is close to 1 . Hence, as $\varepsilon \rightarrow 0$, we have that

$$
\frac{f^{\prime}(\varepsilon)}{\left(\frac{\left\|y_{\varepsilon}\right\|}{\cos \theta_{\varepsilon}}\right)} \longrightarrow 1 \text {. }
$$

Using Theorems 2 and 4 , one can also find a formula for the $\delta_{\varepsilon}$ introduced in Theorem 2.

Theorem 5. Using the notation in Theorem 2, we have

$$
\delta_{\varepsilon}=\frac{f^{\prime}(\varepsilon) f(\varepsilon)}{\varepsilon}, \text { where } f(\varepsilon)=\left\|y_{\varepsilon}\right\| .
$$

Proof. Since $y_{\varepsilon}=\delta_{\varepsilon} T^{*}\left(T y_{\varepsilon}-x_{0}\right)$, we have $\left\langle y_{\varepsilon}, z\right\rangle=\delta_{\varepsilon}\left\langle T y_{\varepsilon}-x_{0}, T z\right\rangle$ for all $z \in H$. Let $z=y_{\varepsilon}$. Then $\left\langle y_{\varepsilon}, y_{\varepsilon}\right\rangle=\delta_{\varepsilon}\left\langle T y_{\varepsilon}-x_{0}, T y_{\varepsilon}\right\rangle$, hence

$$
\begin{gathered}
\delta_{\varepsilon}=\frac{\left\|y_{\varepsilon}\right\|^{2}}{\left\langle T y_{\varepsilon}-x_{0}, T y_{\varepsilon}\right\rangle}=\frac{\left\|y_{\varepsilon}\right\|^{2}}{\left\|T y_{\varepsilon}\right\|\left\|T y_{\varepsilon}-x_{0}\right\| \cos \theta_{\varepsilon}} \\
=\frac{\left\|y_{\varepsilon}\right\|^{2}}{\varepsilon\left\|T y_{\varepsilon}\right\| \cos \theta_{\varepsilon}}=\frac{f^{\prime}(\varepsilon) f(\varepsilon)}{\varepsilon},
\end{gathered}
$$

where $f(\varepsilon)=\left\|y_{\varepsilon}\right\|$.

The next result is an improvement of Theorem 3 from 11. This theorem was proved for a subsequence of a sequence $\left(\varepsilon_{n}\right)=\left(\varepsilon_{n-1} \sin \theta_{n-1}\right)$, where $\theta_{n-1}=\theta_{\varepsilon_{n-1}}$ is the angle between the vectors $T y_{\varepsilon_{n-1}}-x_{0}$ and $T y_{\varepsilon_{n-1}}$ and the $\varepsilon_{n}$ 's are radii with $\varepsilon_{n} \rightarrow 0$. We will show that the theorem remains true for any sequence of $\varepsilon_{n}$ 's with $\varepsilon_{n} \rightarrow 0$.

Theorem 6. For any bounded operator $T$ with dense range, if $x_{0} \notin R(T)$, then

$$
\frac{1}{\varepsilon}\left\langle T y_{\varepsilon}-x_{0}, T y_{\varepsilon}\right\rangle \longrightarrow 0 \quad \text { as } \varepsilon \rightarrow 0
$$


Proof. Let $\left(\varepsilon_{n}\right)$ be a sequence with $\varepsilon_{n} \rightarrow 0$. In Theorem 3 of 11 it is proved that if $\varepsilon_{n}=\varepsilon_{n-1} \sin \theta_{n-1}$, where $\theta_{n-1}$ is the angle between the vectors $T y_{\varepsilon_{n-1}}-x_{0}$ and $T y_{\varepsilon_{n-1}}$, then there is a subsequence $\left(n_{k}\right)$ of natural numbers such that $\theta_{n_{k}} \longrightarrow \frac{\pi}{2}$. Consequently, $\cos \theta_{n_{k}} \longrightarrow 0$ for the same subsequence. From the definition of $y_{\varepsilon}$ we also know that $\left\|y_{\varepsilon}\right\| \rightarrow \infty$ as $\varepsilon \rightarrow 0$. By Theorem 4 we have that the size of the derivative $f^{\prime}\left(\varepsilon_{n_{k}}\right)$ is asymptotically the same as the size of $\frac{\left\|y_{\varepsilon_{n_{k}}}\right\|}{\cos \theta_{n_{k}}}$, so $\left|f^{\prime}\left(\varepsilon_{n_{k}}\right)\right|$ is increasing as a function of $k$ as $k \rightarrow \infty$. Since, by Theorem 3 , the function $f(\varepsilon)=\left\|y_{\varepsilon}\right\|$ is convex on $\left(0,\left\|x_{0}\right\|\right)$, we have that $\left|f^{\prime}\left(\varepsilon_{n}\right)\right|$ is increasing as $n \rightarrow \infty$ for all sequences $\varepsilon_{n}$, where $\varepsilon_{n} \rightarrow 0$. We will prove that $\theta_{n} \longrightarrow \frac{\pi}{2}$ as $n \rightarrow \infty$. If not, then there exists a sequence $\left(r_{n}\right)$ with $r_{n} \rightarrow 0$ and $\sum r_{n}<\infty$, but $\theta_{r_{n}}>\frac{\pi}{2}+\delta$ for some $\delta>0$ and for all $n \geq n_{0}$. If $\theta_{r_{0}}$ is the angle between the vectors $T y_{r_{0}}-x_{0}$ and $T y_{r_{0}}$, then there exists $t>0$ such that $\left\|x_{0}-(1+t) T y_{r_{0}}\right\|=\varepsilon_{1}$. One can show that $t<K \cdot r_{0}$ for some constant $K>0$. To see this note that $(1+t)\left\|T y_{r_{0}}\right\| \leq$ $\left\|T y_{r_{0}}\right\|+2 r_{0}$, so $t\left\|T y_{r_{0}}\right\| \leq 2 r_{0}$ and $t \leq \frac{2 r_{0}}{\left\|x_{0}\right\|-r_{0}}<K \cdot r_{0}$ for some $K>0$. Thus, by the minimality of $\left\|y_{\varepsilon_{1}}\right\|$, we have $\left\|y_{\varepsilon_{1}}\right\| \leq(1+t)\left\|y_{r_{0}}\right\|<\left(1+K r_{0}\right)\left\|y_{r_{0}}\right\|$. Let $r_{1}, r_{2}, \ldots, r_{k_{1}}$ be $k_{1}$ terms of the sequence $\left(r_{n}\right)$ such that $r_{0}>r_{1}>r_{2}>\cdots>$ $r_{k_{1}} \geq \varepsilon_{1}$. By Definition 1 we also have that $\left\|y_{r_{0}}\right\| \leq\left\|y_{r_{1}}\right\| \leq\left\|y_{r_{2}}\right\| \leq \ldots \leq\left\|y_{r_{k_{1}}}\right\| \leq$ $\left\|y_{\varepsilon_{1}}\right\|<\left(1+K r_{0}\right)\left\|y_{r_{0}}\right\|$. For $n \geq 2$ let $\varepsilon_{n}=\varepsilon_{n-1} \cdot \sin \theta_{n-1}$. Let $r_{k_{n-1}+1}, \ldots, r_{k_{n}}$ be the terms from the sequence $\left(r_{n}\right)$ such that $r_{k_{n-1}+1}>r_{k_{n-1}+2}>\cdots>r_{k_{n}} \geq \varepsilon_{n}$. Applying the above argument repeatedly, we get for $n \geq 2$ and $k_{n-1}<m \leq k_{n}$ that $\left\|y_{r_{m}}\right\| \leq\left\|y_{\varepsilon_{n}}\right\| \leq\left(1+K \varepsilon_{0}\right)\left(1+K \varepsilon_{1}\right) \ldots\left(1+K \varepsilon_{n}\right)\left\|y_{r_{0}}\right\|$. Since $\sum \varepsilon_{n}<\infty$, the infinite product $\left(1+K \varepsilon_{0}\right)\left(1+K \varepsilon_{1}\right) \ldots=M$ is finite, and so $\left\|y_{r_{n}}\right\| \leq M\left\|y_{r_{0}}\right\|$ for all $n$. Let $\left(y_{n_{k}}\right)$ be a weakly convergent subsequence, converging weakly to $y_{0}$. Then, $T y_{n_{k}} \stackrel{w}{\longrightarrow} T y_{0}$ and $\left\|T y_{n_{k}}-x_{0}\right\|=r_{n_{k}} \longrightarrow 0$. So, $\left\|T y_{0}-x_{0}\right\|=0$, that is, $T y_{0}=x_{0}$. This contradiction proves that $\theta_{\varepsilon_{n}} \longrightarrow \frac{\pi}{2}$ as $\varepsilon_{n} \rightarrow 0$ for any sequence of $\varepsilon_{n}$ 's.

At this point we do not know whether Theorem 5 from 1 holds for all $n$. This theorem says that if a quasinilpotent operator $T$ has no hyperinvariant subspaces, then there is a subsequence $\left(n_{k}\right)$ of natural numbers such that for some $\delta>0$ we have

$$
\left\langle T^{n_{k}} y_{n_{k}}^{\delta}, T^{n_{k}} y_{n_{k}}^{\delta}-x_{0}\right\rangle \longrightarrow 0 .
$$

We can, however, give the following improvement.

Proposition 7. Let $T$ be a quasinilpotent operator. Let $x_{0} \in H$. Then there is a subsequence $\left(n_{k}\right)$ of natural numbers such that for some $\delta>0$ and for every $m \in \mathbb{N}$ we have

$$
\left\langle T^{n_{k}+m} y_{n_{k}+m}^{\delta}, T^{n_{k}+m} y_{n_{k}+m}^{\delta}-x_{0}\right\rangle \longrightarrow 0
$$

Proof. The proposition says that there are infinitely many such subsequences $\left(n_{k}\right)$ with the property (2). To see this, for any $m=1,2,3, \ldots$, find $l_{m}(T)$ with $\left\|l_{m}(T)\right\| \leq C$ such that $\left\|T^{m} l_{m}(T) x_{0}-x_{0}\right\|<\frac{\delta}{2}$. Here, $l_{m}(T)$ denotes a polynomial in $T$. Let $\left\|T^{n_{k}} y_{n_{k}}-x_{0}\right\| \leq \frac{\delta}{2 C}$. Then, for any fixed $m=1,2,3, \ldots$, we have

$$
\begin{gathered}
\left\|T^{n_{k}+m} l_{m}(T) y_{n_{k}}-x_{0}\right\| \leq\left\|T^{n_{k}+m} l_{m}(T) y_{n_{k}}-l_{m}(T) T^{m} x_{0}\right\|+\left\|l_{m}(T) T^{m} x_{0}-x_{0}\right\| \\
\leq\left\|T^{m} l_{m}(T)\right\|\left\|T^{n_{k}} y_{n_{k}}-x_{0}\right\|+\frac{\delta}{2} \leq\left\|T^{m}\right\| C \cdot \frac{\delta}{2 C}+\frac{\delta}{2}=\left(\left\|T^{m}\right\|+1\right) \frac{\delta}{2}=\delta
\end{gathered}
$$

assuming that $\|T\|=1$. 
The minimality of $y_{n_{k}+m}^{\delta}$ gives

$$
\left\|y_{n_{k}+m}^{\delta}\right\| \leq\left\|l_{m}(T) y_{n_{k}}^{\frac{\delta}{2 C}}\right\| \leq C\left\|y_{n_{k}}^{\frac{\delta}{2 C}}\right\| .
$$

Thus, the ratio $\left\|y_{n_{k}+m}^{\delta}\right\| /\left\|y_{n_{k}}^{\frac{\delta}{2 C}}\right\|$ is bounded by a constant. Now take $\varepsilon<\frac{\delta}{2 C}$. Since the function $f(\varepsilon)=\left\|y_{\varepsilon}\right\|$ is convex, and since the modulus of its derivative goes to infinity as $\varepsilon \rightarrow 0$, we have $\frac{\left\|y_{n_{k}}^{\varepsilon}\right\|}{\left\|y_{n_{k}}^{\frac{2}{2}}\right\|} \longrightarrow \infty$ as $\varepsilon \rightarrow 0$. From this it follows that for every $\varepsilon>0, \theta_{n_{k}}^{\varepsilon} \longrightarrow \frac{\pi}{2}$ as $k \rightarrow \infty$. As mentioned above, since $\left\|y_{n_{k}+m}^{\delta}\right\|$ differs from $\left\|y_{n_{k}}^{\frac{\delta}{2 C}}\right\|$ only by a constant, we also have that for every $\varepsilon<\frac{\delta}{2 C}, \frac{\left\|y_{n_{k}}^{\varepsilon}\right\|}{\left\|y_{n_{k}+m}^{\delta}\right\|} \longrightarrow \infty$ as $k \rightarrow \infty$. Since $\left\|y_{n_{k}+m}^{\varepsilon}\right\| \geq\left\|y_{n_{k}}^{\varepsilon}\right\|$, we get $\frac{\left\|y_{n_{k}+m}^{\varepsilon}\right\|}{\left\|y_{n_{k}+m}^{\delta}\right\|} \longrightarrow \infty$ as $k \rightarrow \infty$. Hence, on the sphere with radius $\varepsilon$, if we have a subsequence $\left(n_{k}\right)$ for which $\theta_{n_{k}}^{\varepsilon} \longrightarrow \frac{\pi}{2}$ as $k \rightarrow \infty$, then, in fact, $\theta_{n_{k}+m}^{\varepsilon} \longrightarrow \frac{\pi}{2}$ for any $m=1,2,3, \ldots$ as $k \rightarrow \infty$. Hence for every $m \in \mathbb{N}$ we have

$$
\left\langle T^{n_{k}+m} y_{n_{k}+m}^{\delta}, T^{n_{k}+m} y_{n_{k}+m}^{\delta}-x_{0}\right\rangle \longrightarrow 0 .
$$

Suppose $T$ is quasinilpotent and has no hyperinvariant subspaces. Assume $x_{0} \notin$ $R(T)$. Then, by the previous proposition, for some $\varepsilon>0$ and for some subsequence $\left(n_{k}\right)$ of integers,

$$
\left\langle T^{n_{k}} y_{n_{k}}^{\varepsilon}, T^{n_{k}} y_{n_{k}}^{\varepsilon}-x_{0}\right\rangle \longrightarrow 0,
$$

where $\left(y_{n_{k}}^{\varepsilon}\right)$ are the backward minimal vectors with respect to $x_{0}$ and $\varepsilon$. As in [3], we can write (renaming $n=n_{k}$ ) that

$$
T^{n} y_{n, x_{0}}^{\varepsilon}=\left(1-\varepsilon^{2}\right) x_{0}+\gamma \varepsilon \sqrt{1-\varepsilon^{2}} s_{0}+\sqrt{1-\varepsilon^{2}} \varepsilon \sqrt{1-\gamma^{2}} s_{n},
$$

where $\left\|s_{0}\right\|=\left\|s_{n}\right\|=1$ and $s_{n} \stackrel{w}{\rightarrow} 0$.

S.Ansari and P.Enflo have shown that if $T$ is quasinilpotent and has no hyperinvariant subspaces, then for every $x_{0} \in H$ with $\left\|x_{0}\right\|=1$, for every $\varepsilon>0$, and for every $\gamma>\sqrt{2 \varepsilon}$, there are $x_{1}$ with $\left\|x_{1}\right\|=1$ and $\varepsilon_{1}>0$ with $0<\varepsilon_{1}<\varepsilon$ such that $\left\|x_{0}-x_{1}\right\| \leq \varepsilon g(\gamma)$, where $g(\gamma)$ is a continuous function of $\gamma$, and such that for some subsequence $\left(n_{k}\right)$ of integers we have

$$
T^{n_{k}} y_{n_{k}, x_{1}}^{\varepsilon_{1}}=a_{n_{k}} x_{1}+s_{n_{k}},
$$

where $a_{n_{k}}$ is a constant, $s_{n_{k}} \perp x_{1}$, and each weak limit of $s_{n_{k}}$ has norm less than $\gamma \varepsilon_{1}$ (see Theorem 6, 1]).

First we will show how to find $x_{1}$ and then we will determine the function $g(\gamma)$.

Let

$$
x_{1}=\frac{\sqrt{1-\varepsilon^{2}}}{\sqrt{1-\varepsilon^{2}\left(1-\gamma^{2}\right)}} x_{0}+\frac{\gamma \varepsilon}{\sqrt{1-\varepsilon^{2}\left(1-\gamma^{2}\right)}} s_{0}
$$


where $x_{0} \perp s_{0}$, and $\left\|x_{0}\right\|=\left\|s_{0}\right\|=1$ as in (3). Then, clearly, $\left\|x_{1}\right\|=1$, and

$$
\begin{gathered}
\left\|x_{0}-x_{1}\right\|^{2}=\left\|x_{0}-\frac{1-\varepsilon^{2}}{\sqrt{1-\varepsilon^{2}\left(1-\gamma^{2}\right)}} x_{0}-\frac{\gamma \varepsilon}{\sqrt{1-\varepsilon^{2}\left(1-\gamma^{2}\right)}} s_{0}\right\|^{2} \\
=\frac{1}{1-\varepsilon^{2}\left(1-\gamma^{2}\right)}\left(1-\varepsilon^{2}\left(1-\gamma^{2}\right)-2 \sqrt{1-\varepsilon^{2}} \sqrt{1-\varepsilon^{2}\left(1-\gamma^{2}\right)}+1-\varepsilon^{2}+\gamma^{2} \varepsilon^{2}\right) \\
=2\left(1-\frac{\sqrt{1-\varepsilon^{2}}}{\sqrt{1-\varepsilon^{2}\left(1-\gamma^{2}\right)}}\right) \approx 2\left(1-\frac{1-\frac{\varepsilon^{2}}{2}}{1-\frac{\varepsilon^{2}}{2}\left(1-\gamma^{2}\right)}\right) \\
\approx 2\left(1-\left(1-\frac{\varepsilon^{2}}{2}\right)\left(1+\frac{\varepsilon^{2}}{2}\left(1-\gamma^{2}\right)\right)\right)=\varepsilon^{2}\left(1-\left(1-\frac{\varepsilon^{2}}{2}\right)\left(1-\gamma^{2}\right)\right) .
\end{gathered}
$$

The approximation $\approx$ comes from approximating the square root by the first two terms in its power series representation. Thus,

$$
\left\|x_{0}-x_{1}\right\| \approx \varepsilon\left(1-\frac{1}{2}\left(1-\frac{\varepsilon^{2}}{2}\right)\left(1-\gamma^{2}\right)\right)=\varepsilon g(\gamma)<\varepsilon,
$$

where $g(\gamma)=1-\frac{1}{2}\left(1-\frac{\varepsilon^{2}}{2}\right)\left(1-\gamma^{2}\right)$.

Next we derive a formula for $\varepsilon_{1}$.

Proposition 8. Let all the assumptions about $T$ hold as above. Let $x_{0}, \varepsilon$ and $\gamma$ be given. Let $x_{1}$ be defined as in (5). Then $\varepsilon_{1}$ in (4) is given by $\varepsilon_{1}=\varepsilon \sqrt{1-\gamma^{2}}$.

Proof. Let $x_{0} \in H$ with $\left\|x_{0}\right\|=1$. Then, given $\varepsilon>0$ and $\gamma>0$ we can write $T^{n_{k}} y_{n_{k}, x_{0}}^{\varepsilon}=\left(1-\varepsilon^{2}\right) x_{0}+\gamma \varepsilon \sqrt{1-\varepsilon^{2}} s_{0}+\sqrt{1-\gamma^{2}} \varepsilon \sqrt{1-\varepsilon^{2}} s_{n_{k}}$. If $x_{1}$ is as in (5), then, by Theorem 6 in [1], there exists an $\varepsilon_{1}$ with $0<\varepsilon_{1}<\varepsilon$ such that

$$
\begin{aligned}
\left(1-\varepsilon_{1}^{2}\right) x_{1}+\varepsilon_{1} \sqrt{1-\varepsilon_{1}^{2}} s_{n_{k}}=\frac{\sqrt{1-\varepsilon_{1}^{2}}}{\sqrt{1-\varepsilon^{2}}}\left(\left(1-\varepsilon^{2}\right) x_{0}\right. & +\gamma \varepsilon \sqrt{1-\varepsilon^{2}} s_{0} \\
& \left.+\sqrt{1-\gamma^{2}} \varepsilon \sqrt{1-\varepsilon^{2}} s_{n_{k}}\right) .
\end{aligned}
$$

Note that the right-hand side represents a vector that is tangent to the $\varepsilon_{1}$-ball around $x_{1}$. Comparing the ratios of sides of the similar triangles in the plane spanned by $x_{1}$ and $T^{n} y_{n, x_{0}}^{\varepsilon}$, we get

$$
\frac{1-\varepsilon_{1}^{2}}{\varepsilon_{1} \sqrt{1-\varepsilon_{1}^{2}}}=\frac{\sqrt{\left(1-\varepsilon^{2}\right)^{2}+\gamma^{2} \varepsilon^{2}\left(1-\gamma^{2}\right)}}{\sqrt{1-\gamma^{2}} \varepsilon \sqrt{1-\varepsilon^{2}}}
$$

Squaring both sides of (6) we get

$$
\frac{1-\varepsilon_{1}^{2}}{\varepsilon_{1}^{2}}=\frac{\left(1-\varepsilon^{2}\right)\left(1-\varepsilon^{2}+\gamma^{2} \varepsilon^{2}\right)}{\left(1-\varepsilon^{2}\right) \varepsilon^{2}\left(1-\gamma^{2}\right)}=\frac{1-\varepsilon^{2}\left(1-\gamma^{2}\right)}{\varepsilon^{2}\left(1-\gamma^{2}\right)},
$$

where it follows that $\varepsilon_{1}=\varepsilon \sqrt{1-\gamma^{2}}$.

Denote by $A^{\delta_{n}}$ the continuous linear operator

$$
-\delta_{n}\left(I-\delta_{n} T^{n} T^{* n}\right)^{-1} T^{n} T^{* n} .
$$

From the orthogonality equations we obtain that $A^{\delta_{n}} x_{0}=T^{n} y_{n, x_{0}}$, that is, given $x_{0}$, the $\delta_{n}$ determines a backward minimal vector $y_{n, x_{0}}$. Recall that $\delta_{n}$ depends on $\varepsilon$.

The following proposition gives an asymptotic relationship between the norm of $y_{n, x_{0}}$ and the absolute value of $\delta_{n}$. 
Proposition 9. Let $\varepsilon_{2}<\varepsilon_{1}<\varepsilon_{0}$. Then, for $n$ sufficiently large, we have

$$
\left|\delta_{n}\left(\varepsilon_{0}\right)\right| \leq \frac{\left\|y_{n}^{\varepsilon_{1}}\right\|^{2}}{2 \varepsilon_{1}\left(\varepsilon_{0}-\varepsilon_{1}\right)} \leq\left|\delta_{n}\left(\varepsilon_{2}\right)\right| \text {. }
$$

Proof. Integrating $\delta_{n}(\varepsilon)=\frac{f_{n}(\varepsilon) f_{n}^{\prime}(\varepsilon)}{\varepsilon}$ by parts over a small interval $\left[\varepsilon_{1}, \varepsilon_{0}\right]$ (where $f_{n}(\varepsilon)=\left\|y_{n}^{\varepsilon}\right\|$ and $\left.f_{n}^{\prime}(\varepsilon)=\frac{\left\|y_{n}^{\varepsilon}\right\|}{\cos \theta_{n}^{\varepsilon}\left\|T y_{n}^{\varepsilon}\right\|}\right)$, we get

$$
\begin{gathered}
\left|\int_{\varepsilon_{1}}^{\varepsilon_{0}} \varepsilon \delta_{n}(\varepsilon) d \varepsilon\right|=\left|\int_{\varepsilon_{1}}^{\varepsilon_{0}} f_{n}(\varepsilon) f_{n}^{\prime}(\varepsilon) d \varepsilon\right| \\
=\left|f_{n}^{2}(\varepsilon)\right|_{\varepsilon_{1}}^{\varepsilon_{0}}-\left.\int_{\varepsilon_{1}}^{\varepsilon_{0}} f_{n}(\varepsilon) f_{n}^{\prime}(\varepsilon) d \varepsilon|=| \frac{f_{n}^{2}(\varepsilon)}{2}\right|_{\varepsilon_{1}} ^{\varepsilon_{0}}=\left|\frac{f_{n}^{2}\left(\varepsilon_{0}\right)}{2}-\frac{f_{n}^{2}\left(\varepsilon_{1}\right)}{2}\right| .
\end{gathered}
$$

Since $\theta_{n}^{\varepsilon} \longrightarrow \frac{\pi}{2}$ as $n \rightarrow \infty$, by Thorem 4 we know that $\left|f_{n}^{\prime}(\varepsilon)\right| \longrightarrow \infty$ very fast as $\varepsilon \rightarrow 0$ and as $n \rightarrow \infty$. Therefore, the size of the above integral is mostly determined by the $f_{n}$ 's value at $\varepsilon_{1}$. Considering $\varepsilon$ as a constant over the interval $\left[\varepsilon_{1}, \varepsilon_{0}\right]$ (e.g. taking $\left.\varepsilon=\varepsilon_{1}\right)$, we get

$$
\left|\int_{\varepsilon_{1}}^{\varepsilon_{0}} \delta_{n}(\varepsilon) d \varepsilon\right| \approx \frac{f_{n}^{2}\left(\varepsilon_{1}\right)}{2 \varepsilon_{1}} .
$$

Now, for some $\varepsilon_{2}<\varepsilon_{1}<\varepsilon_{0} \quad\left(\varepsilon_{2} \leq \varepsilon_{1}\left(\varepsilon_{0}-\varepsilon_{1}\right)\right)$, we have

$$
\begin{gathered}
\left|\delta_{n}\left(\varepsilon_{0}\right)\right| \leq\left|\delta_{n, \text { aver }\left[\varepsilon_{1}, \varepsilon_{0}\right]}(\varepsilon)\right|=\frac{1}{\varepsilon_{0}-\varepsilon_{1}}\left|\int_{\varepsilon_{1}}^{\varepsilon_{0}} \delta_{n}(\varepsilon) d \varepsilon\right| \leq \frac{1}{\varepsilon_{0}-\varepsilon_{1}} \cdot \frac{f_{n}^{2}\left(\varepsilon_{1}\right)}{2 \varepsilon_{1}} \\
\leq \frac{f_{n}^{2}\left(\varepsilon_{2}\right)}{\varepsilon_{2}} \leq\left|\int_{\varepsilon_{2}}^{\varepsilon_{1}} \delta_{n}(\varepsilon) d \varepsilon\right| \leq \frac{1}{\varepsilon_{1}-\varepsilon_{2}}\left|\int_{\varepsilon_{2}}^{\varepsilon_{1}} \delta_{n}(\varepsilon) d \varepsilon\right|=\left|\delta_{n, \text { aver }\left[\varepsilon_{2}, \varepsilon_{1}\right]}(\varepsilon)\right| \leq\left|\delta_{n}\left(\varepsilon_{2}\right)\right| .
\end{gathered}
$$

Hence, for $n$ large enough, we have

$$
\left|\delta_{n}\left(\varepsilon_{0}\right)\right| \leq \frac{f_{n}^{2}\left(\varepsilon_{1}\right)}{2 \varepsilon_{1}\left(\varepsilon_{0}-\varepsilon_{1}\right)} \leq\left|\delta_{n}\left(\varepsilon_{2}\right)\right| .
$$

It follows from Theorem 5 and Proposition 7 that for some subsequence $\left(n_{k}\right)$, if $\varepsilon_{2}<\varepsilon_{1}$, then

and if $\varepsilon_{0}>\varepsilon_{1}$, then

$$
\frac{\left|\delta_{n_{k}}\left(\varepsilon_{2}\right)\right|}{\left|\delta_{n_{k}}\left(\varepsilon_{1}\right)\right|} \longrightarrow \infty \text { as } n_{k} \rightarrow \infty
$$

$$
\frac{\left|\delta_{n_{k}}\left(\varepsilon_{0}\right)\right|}{\left|\delta_{n_{k}}\left(\varepsilon_{1}\right)\right|} \longrightarrow 0 \text { as } n_{k} \rightarrow \infty .
$$

Combining this with Proposition 9, we get the following theorem:

Theorem 10. Let $\varepsilon_{1}>0$. There is a subsequence $\left(n_{k}\right)$ of natural numbers such that for every $\varepsilon_{2}<\varepsilon_{1}$ we have

$$
\frac{\left|\delta_{n_{k}}\left(\varepsilon_{2}\right)\right|}{\left\|y_{n_{k}}^{\varepsilon_{1}}\right\|^{2}} \longrightarrow \infty \text { as } k \rightarrow \infty,
$$

and for every $\varepsilon_{0}>\varepsilon_{1}$ we have

$$
\frac{\left|\delta_{n_{k}}\left(\varepsilon_{0}\right)\right|}{\left\|y_{n_{k}}^{\varepsilon_{1}}\right\|^{2}} \longrightarrow 0 \text { as } k \rightarrow \infty
$$


Corollary 11. If $\left|\delta_{n_{k}}\left(\mu_{k}\right)\right|=\left\|y_{n_{k}, x_{0}}^{\varepsilon_{k}}\right\|^{2}$ for all $k$ large enough, then

$$
\lim _{k} \sup \mu_{k}=\varepsilon=\lim _{k} \sup \varepsilon_{k} .
$$

Proof. WLOG suppose $\lim _{k} \sup \mu_{k}=\varepsilon$, but $\lim _{k} \sup \varepsilon_{k} \neq \varepsilon$ with $\varepsilon_{k}<\varepsilon$. Then there exist a $\delta>0$ and $k_{0} \in \mathbb{N}$ such that $\varepsilon_{k}<\varepsilon-\delta$ for all $k>k_{0}$. By Theorem 10 we have

$$
\frac{\left|\delta_{n_{k}}\left(\mu_{k}\right)\right|}{\left\|y_{n_{k}}^{\varepsilon_{k}}\right\|^{2}} \leq \frac{\left|\delta_{n_{k}}\left(\mu_{k}\right)\right|}{\left\|y_{n_{k}}^{\varepsilon-\delta}\right\|^{2}} \longrightarrow 0
$$

as $k \rightarrow \infty$, contradicting the assumption that $\left|\delta_{n_{k}}\left(\mu_{k}\right)\right|=\left\|y_{n_{k}, x_{0}}^{\varepsilon_{k}}\right\|^{2}$ for all $k$ large enough.

Now, having $x_{0}$ and $\varepsilon$ fixed, we get $\delta_{n_{k}}$ and apply the operator $A^{\delta_{n_{k}}}$ from (7) to the vector $x_{1}$. We know that $\left\|A^{\delta_{n_{k}}} x_{0}-x_{0}\right\|=\left\|T^{n_{k}} y_{n_{k}, x_{0}}^{\varepsilon}-x_{0}\right\|=\varepsilon$. We will show that when $x_{0}$ is replaced by $x_{1}$, we get a better approximation.

Proposition 12. With the notation as above,

$$
\lim _{k} \sup \left\|A^{\delta_{n_{k}}} x_{1}-x_{1}\right\| \leq \varepsilon \sqrt{1-\gamma^{2}} .
$$

Proof. Let $x_{0}$ and $\varepsilon$ be given. For every $k \in \mathbb{N}$ find an $\varepsilon_{k}<\varepsilon$ such that $\left|\delta_{n_{k}, x_{0}}^{\varepsilon}\right|=$ $\left\|y_{n_{k}, x_{0}}^{\varepsilon_{k}}\right\|^{2}$. As a special case of Corollary 11, we have that $\varepsilon_{k} \rightarrow \varepsilon$. Let $x_{1}$ be as in (5). Then, if $\left\|y_{n_{k}, x_{0}}^{\varepsilon_{k}}\right\|=\left\|y_{n_{k}, x_{1}}^{v_{k}}\right\|$ for some sequence $\left(v_{k}\right)$ and for all $k$, then, by Proposition $8, \lim _{k} \sup v_{k} \leq \varepsilon \sqrt{1-\gamma^{2}}$. On the other hand, $\left\|y_{n_{k}, x_{1}}^{v_{k}}\right\|^{2}=$ $\left|\delta_{n_{k}, x_{1}}^{\mu_{k}}\right|$ for some sequence $\left(\mu_{k}\right)$ with $\lim _{k} \sup \mu_{k}=\lim _{k} \sup v_{k} \leq \varepsilon \sqrt{1-\gamma^{2}}$. Hence, $\lim _{k} \sup \left\|T^{n_{k}} y_{n_{k}, x_{1}}^{v_{k}}-x_{1}\right\| \leq \varepsilon \sqrt{1-\gamma^{2}}$.

We now start over with $x_{1}$ instead of $x_{0}$ and $\varepsilon_{1}$ instead of $\varepsilon$. Similarly to (5), for every $\gamma_{1}>\sqrt{2 \varepsilon_{1}}$, we define

$$
x_{2}=\frac{\sqrt{1-\varepsilon_{1}^{2}}}{\sqrt{1-\varepsilon_{1}^{2}\left(1-\gamma_{1}^{2}\right)}} x_{1}+\frac{\gamma_{1} \varepsilon_{1}}{\sqrt{1-\varepsilon_{1}^{2}\left(1-\gamma_{1}^{2}\right)}} s_{1} .
$$

Then

$$
\left\|x_{1}-x_{2}\right\| \approx \varepsilon\left(1-\frac{\gamma^{2}}{2}\right)\left(1-\frac{1}{2}\left(1-\frac{\varepsilon^{2}}{2}\left(1-\gamma^{2}\right)\right)\left(1-\gamma_{1}^{2}\right)\right) .
$$

Also,

$$
\left\|x_{0}-x_{2}\right\| \approx \varepsilon\left(1-\frac{1}{2}\left(1-\gamma^{2}\right)\left(\left(1-\gamma_{1}^{2}\right)\left(1-\frac{\varepsilon^{2}}{2}\left(1-\gamma^{2}\right)\right)-\frac{\varepsilon^{2}}{2}\right)\right) .
$$

Similarly to Proposition 8, we get that $\varepsilon_{2}=\varepsilon_{1} \sqrt{1-\gamma^{2}}=\varepsilon\left(1-\gamma^{2}\right)$, and we repeat previous steps with $x_{3}, \varepsilon_{2}$ and $\gamma_{2}>\sqrt{2 \varepsilon_{2}}$ instead.

In general, we define

$$
x_{n+1}=\frac{\sqrt{1-\varepsilon_{n}^{2}}}{\sqrt{1-\varepsilon_{n}^{2}\left(1-\gamma_{n}^{2}\right)}} x_{n}+\frac{\gamma_{n} \varepsilon_{n}}{\sqrt{1-\varepsilon_{n}^{2}\left(1-\gamma_{n}^{2}\right)}} s_{n},
$$

from where it follows that

$$
\left\|x_{n+1}-x_{n}\right\| \approx \varepsilon \prod_{k=0}^{n-1}\left(1-\frac{\gamma_{k}^{2}}{2}\right)\left(\gamma^{2}+\frac{\varepsilon^{2}}{2} \prod_{k=0}^{n}\left(1-\gamma_{k}^{2}\right)\right) .
$$


Remark. We conjecture that if the sequence $\left(x_{n}\right)$ converges and the $\varepsilon_{n}$ 's go to zero, then the limit vector is noncyclic. For the further use of extremal vectors it is of interest to know whether the sequence $\left(x_{n}\right)$, that is defined inductively as in (8), always converges or whether it may go weakly to zero.

\section{REFERENCES}

1. S. Ansari, P. Enflo, Extremal vectors and invariant subspaces, Trans. Amer. Math. Soc. 350 (1998), 539-558. MR 98d:47019

2. P. Enflo, Extremal vectors for a class of linear operators, Functional Analysis and Economic Theory, Springer-Verlag (1998), 61-64. MR 2000k:47020

3. P. Enflo, V. Lomonosov, Some aspects of the Invariant Subspace Problem, to appear.

Department of Mathematics, Kent State University, Kent, Ohio 44242

E-mail address: enflo@mcs.kent.edu

Department of Mathematics, Kent State University, Kent, Ohio 44242

E-mail address: terje.hoim@trincoll.edu 